\title{
Fever episodes in early life and the development of atopy in children with asthma
}

\author{
M. Calvani Jr*, C. Alessandri*, E. Bonci\#
}

Fever episodes in early life and the development of atopy in children with asthma. M. Calvani Jr, C. Alessandri, E. Bonci. C)ERS Journals Ltd 2002.

ABSTRACT: The hygiene hypothesis proposes that declining exposure to infections may contribute to the allergy and asthma "epidemic", but studies to identify the infections involved have so far provided conflicting data. An alternative approach could be based on an attempt to identify the mechanisms common to several different infections, which may exert an atopy "protective" effect. One of these mechanisms may be fever, which is frequently associated with infections and may stimulate the synthesis of interferon- $\gamma$. The aim of this study was to test whether the frequency of fever episodes in the first years of life are related to the development of atopy.

Asthmatic (cases, $n=317$ ) and nonasthmatic children (controls, $n=304$ ) attending the outpatients' allergy clinic were enrolled. The number of fever episodes in the first 2 yrs of life was retrospectively assessed by parental report via a questionnaire completed by interview. Atopy was determined by skin-prick tests to 10 prevalent inhalant allergens at the time of examination.

Fever episodes in the first or in the second year of life were significantly more frequent among children with asthma. However, this difference was totally accounted for by a subgroup of children who started wheezing in the first 12 months of life. A significant inverse association between fever episodes in the first $\mathbf{1 2}$ months of life and atopy was found among cases, but not in controls. The association persisted after adjusting for age, sex, daycare attendance and family history of atopic disease and this was totally accounted for by asthmatic children who started wheezing after the first 12 months of life. No significant association was found between fever episodes in the second year of life and atopy for cases and controls.

The data presented here suggests that fever episodes early in life may affect the natural history of asthma by preventing the development of atopy and highlights the need to monitor fever episodes of any kind in longitudinal studies aimed at investigating the hygiene hypothesis prospectively.

Eur Respir J 2002; 20: 391-396.
*Dept of Paediatrics, San Camillo De Lellis Hospital, and "\#pept of Paediatrics, Policlinico Umberto I, Rome, Italy.

Correspondence: M. Calvani Jr

Via Mar della Cina 200

00144

Rome

Italy

Fax: 390686320424

E-mail: MI5660@mclink.it

Keywords: Asthma

atopy

children

fever

infection

Received: August 202001

Accepted after revision: February 28 2002
The reasons why allergy and asthma have increased in Western countries are still unknown. A number of studies suggested that this trend may be caused by a lower exposure to microbes and declining family size ("hygiene hypothesis") [1]. The prevalence of atopy was found to be lower among children of large families [2] or children attending daycare centres [3, 4], two markers of higher exposure to infections. Other studies reported a lower prevalence of atopy or atopic diseases among children exposed to measles [5], hepatitis A [6] or tuberculosis [7], even though the conclusion of this last study has been disputed [8]. The occurrence of uncomplicated common colds (runny nose) in the first year of life [9], specific infections $[5,7]$, frequent respiratory infections [10], or even continuous immune stimulation due to a high turnover of the intestinal flora [11] have been reported to have a protective effect. In contrast, other epidemiological studies did not support the hygiene hypothesis [12] or the alleged atopy-protective role of specific infections such as measles [13], or have even suggested that infections may favour the development of asthma and allergic sensitisations [14]. Therefore, the attempts to identify which infections may protect from the development of atopy or asthma have so far been inconclusive [15].

Another approach to the hygiene hypothesis is based on identifying mechanisms common to several different infections, that may exert an atopy "protective" effect. Fever is frequently associated with infections in the first years of life and it is known that physiological mild hyperthermia may stimulate synthesis of interferon (IFN)- $\gamma$ [16]. Interestingly, vON Mutius et al. [17] found that the risk of being atopic was inversely related to the number of fever episodes in the first year of life only among students with asthma examined in an epidemiological setting. The aim of this study was to verify if this association could be observed in a clinical setting. Therefore, the relationship between the number of fever episodes early in life and atopy, among >300 asthmatic children and a group of children who had never reported wheeze or asthma, was examined retrospectively. 


\section{Materials and methods}

\section{Study design and subjects}

This was a case-controlled study enrolling 621 children (36 months to 15 yrs of age) attending the outpatient allergy clinic (Dept of Paediatrics, San Camillo de Lellis Hospital, Rome, Italy) for the first time during the period January 1999-March 2001. Patients were primarily from the middle to lower socioeconomic classes and most were residents within the city. Exclusion criteria were immunodeficiency or connective tissue disease.

Information regarding asthma was obtained by the following questions: 1) How many wheezing episodes ("bronchite asmatiforme o broncospasmo") has your child had?; 2) At what age did this first happen?; 3) Did your doctor ever define your child as being affected by asthma?; and 4) Did your doctor ever prescribe $\beta_{2}$-agonist therapy? Atopic dermatitis was defined as flexural, itching dermatitis, as characterised by HANIFIN and RAJKA [18].

Children with a history of $\geqslant 3$ episodes of wheezing requiring $\beta_{2}$-agonist therapy or children diagnosed as being affected by asthma by their doctor (or both) were classified as having asthma $(n=317)$; this category was further divided into three groups, those who started wheezing during the first year of life (group A), during the second year of life (group B), or later (group C).

Children who had never had asthma or wheeze, were included in the control group $(n=304)$. The children in the control group were attending the outpatients' clinic because they were affected by respiratory symptoms (cough, rhinitis, etc) $(59.3 \%)$, atopic dermatitis $(16.8 \%)$, and other gastrointestinal disorders (diarrhoea, failure to thrive, constipation) $(18.1 \%)$ or skin disease (urticaria, eczema, dermatitis) $(9 \%)$.

Parents were invited to answer a questionnaire, administered by interview, including; demographic questions (e.g. number of siblings), passive smoke exposure, pets, age of entry to nursery care, breastfeeding, and weaning. Family histories were considered positive for atopy if the father or the mother had ever had asthma, atopic dermatitis, or allergic rhinitis. Information on fever in the first 2 yrs of life was obtained by the following questions: 1) How many fever episodes $\left(>37^{\circ} \mathrm{C}\right)$ did your child have during the first 12 months of life?; 2) How many fever episodes $\left(>37^{\circ} \mathrm{C}\right)$ did your child have between the 13 th and the 24 th month? All of the above was carried out prior to conducting the skin-prick tests.

\section{Skin-prick tests}

Skin-prick tests were performed on the volar aspects of the forearm with 10 common standardised allergen extracts (Dermatophagoides pteronissinus, $D$. farinae, Alternaria tenuis, Aspergillus fumigatus, Cladosporium herbarum, mixed grass pollen, Artemisia vulgaris, Parietaria officinalis, Olea europaea, cat dander; Bayer ${ }^{\mathrm{TM}}$, Anthony, Cedex, France), using a lancet with a 1-mm tip. Positive (histamine
$10 \mathrm{mg} \cdot \mathrm{mL}^{-1}$ ) and negative (diluent) controls were added. Children with a weal reaction $\geqslant 3 \mathrm{~mm}$ to one or more of the allergens tested $15 \mathrm{~min}$ after the application of the allergen extracts, after subtraction of their reaction to the negative control, were considered atopic.

\section{Statistical analyses}

The Chi-squared test was used for comparisons between groups and an unpaired t-test for comparisons between means. To assess the risk of atopy with repeated fever episodes, the crude odds ratio (OR) was calculated. Adjusted odds ratio (aOR) with 95\% confidence intervals $(95 \% \mathrm{CI})$ were calculated using a logistic regression model performed while adjusting for the covariates that were significantly $(\mathrm{p}<0.05)$ associated with atopy or infection (age, sex, daycare attendance and parental atopy). A p-value of $<0.05$ (two tailed) was considered statistically significant for tests.

\section{Results}

\section{Fever episodes}

The number of fever episodes was known in 581 of $621(93.6 \%)$ and 584 of $621(94 \%)$ children for the first 12 months of life and the second year of life, respectively, without significant differences in the response rate between cases and controls. Fever episodes in the first or in the second year of life were significantly more frequent among children with asthma (group $\mathrm{A}+\mathrm{B}+\mathrm{C}$ ) than controls (table 1). However, this difference was totally accounted for by subjects in group A. In fact, within the asthma groups, fever episodes early in life were significantly more frequent among children who started wheezing in the first year of life compared to the others (table 2), while there was no statistically significant difference in the number of fever episodes between children who started wheezing after the first year of life (group B and C) and controls.

Attending daycare in the first 12 months of life significantly increased the frequency of fever episodes in both cases and in controls; for example, children who attended daycare in the first 12 months of life had an approximately three-times higher risk of being affected from at least five episodes of fever in the first 12 months of life (OR 2.75; 95\% CI 1.16-6.47 in cases; and OR 3.37; 95\% CI 1.12-10.13 in control). The same figures were observed when the number of fever episodes occurring in the first $2 \mathrm{yrs}$ of life were considered (data not shown).

\section{Atopy}

Atopy, defined as skin sensitisation to at least one airborne allergen, was significantly more frequent in cases than in controls (55.2\% versus $38.5 \%$ ) (table 1$)$. The three asthmatic groups were homogeneous for parental atopy and atopic dermatitis. Atopy was more frequent in group B $(59.1 \%)$ and group C $(63.8 \%)$ 
Table 1. - Characteristics of examined outpatients with (cases) and without (controls) asthma

\begin{tabular}{|c|c|c|c|c|}
\hline & Cases & $\%$ & Controls & $\%$ \\
\hline Subjects n & 317 & & 304 & \\
\hline Age yrs & $5.91 \pm 2.78$ & & $6.24 \pm 2.78$ & \\
\hline Males & 202 & 63.7 & 176 & 57.9 \\
\hline Atopy & $175^{* * *}$ & 55.2 & 117 & 38.5 \\
\hline SPT+ve to Dermatophagoides & $133^{* * *}$ & 42.0 & 82 & 27.0 \\
\hline Daycare in the first 12 months & 30 & 9.6 & 22 & 7.4 \\
\hline Parental atopy & 137 & 44.6 & 117 & 39.8 \\
\hline Smoke & 179 & 57.2 & 177 & 59.2 \\
\hline \multicolumn{5}{|l|}{ Older children $n$} \\
\hline 0 & 182 & 59.5 & 182 & 64.5 \\
\hline 1 & 105 & 34.3 & 84 & 29.8 \\
\hline 2 & 16 & 5.2 & 14 & 5 \\
\hline 3 & 3 & 1 & 2 & 0.7 \\
\hline \multicolumn{5}{|l|}{ Fever episodes $n$} \\
\hline $0-12$ months & $2.55 \pm 2.28 * *$ & & $2.11 \pm 2.11$ & \\
\hline 13-24 months & $4.37 \pm 2.64 * * *$ & & $3.66 \pm 2.49$ & \\
\hline $0-24$ months & $6.93 \pm 4.32 * * *$ & & $5.74 \pm 4.17$ & \\
\hline
\end{tabular}

SPT: skin-prick test. ${ }^{* *}: \mathrm{p}=0.01 ; * * * \mathrm{p}<0.001$.

compared to group A (48\%). However, these apparent differences were not statistically significant in the logistical regression adjusted for age (aOR $0.8 ; 95 \%$ CI 0.5-1.3; $\mathrm{p}=0.4$; group A versus $\mathrm{B})$ (aOR 0.7; 95\% CI $0.4-1.2 ; \mathrm{p}=0.3$; group A versus $\mathrm{C}$ ). Sensitisation to Dermatophagoides, the most frequent in the study population, contributed significantly to the observed association between atopy and asthma (table 2). The high prevalence of allergic sensitisation in controls $(38.5 \%)$ is due to the fact that many of them were affected by allergic (nonwheezing) complaints. Having attended daycare in the first 12 months of life did not seem to protect cases or controls from atopic sensitisation (data not shown).

Association between fever episodes and atopic
sensitisations

Among patients with asthma there was a significant inverse association between the number of fever episodes in the first 12 months of life and atopy; this association persisted after adjusting for age, sex, parental atopy, and daycare attendance in the first year of life $(\mathrm{aOR}=0.30 ; 95 \% \mathrm{CI} 0.14-0.64)$. The risk of atopy was approximately three-fold lower among asthmatic children with at least five fever episodes in the first year of life. The higher the number of fever episodes in the first year of life, the lower the risk of being atopic among children with asthma (OR 0.87; 95\% CI 0.77-0.98 per fever episode). An even stronger significant inverse association was observed between fever episodes in the first 12 months of life and sensitisation to Dermatophagoides (fig. 1): only 11 of $48(22.9 \%)$ children with at least five fever episodes were sensitised to Dermatophagoides versus 112 of $252(44.4 \%)$ children with four or less febrile episodes (OR 0.37; 95\% CI 0.18-0.76; $\mathrm{p}=0.005$ ).

Only a weak, nonsignificant inverse association between the number of fever episodes in the first 12 months of life and skin test sensitisations was found in the control group (aOR 0.67; 95\% CI 0.27-1.65).

Table 2. - Characteristics and main background variables for the outpatient with asthma, after subdividing into the three groups

\begin{tabular}{|c|c|c|c|c|c|c|}
\hline & Group A & $\%$ & Group B & $\%$ & Group C & $\%$ \\
\hline Onset of asthma & Before 12 months & & After 12 months & & After 24 months & \\
\hline Subjects $\mathrm{n}$ & 127 & & 186 & & 129 & \\
\hline Age yrs & $5.20 \pm 2.33$ & & $6.37 \pm 2.95^{* * *}$ & & $6.83 \pm 3.08 * * *$ & \\
\hline Males & 76 & 59.8 & 123 & 66.1 & 88 & 67.7 \\
\hline Atopy & 61 & 48 & 110 & 59.1 & $82 * *$ & 63.6 \\
\hline SPT+ve to Dermatophagoides & 41 & 32.3 & $88 * * *$ & 47.3 & $65^{* * *}$ & 50.4 \\
\hline \multicolumn{7}{|l|}{ Fever episodes $\mathrm{n}$} \\
\hline $0-12$ months & $3.13 \pm 2.43$ & & $2.12 \pm 2.02 * * *$ & & $2.01 \pm 1.94 * * *$ & \\
\hline 13-24 months & $5.08 \pm 2.60$ & & $3.86 \pm 2.53^{* * *}$ & & $3.68 \pm 2.55^{* * *}$ & \\
\hline $0-24$ months & $8.22 \pm 4.35$ & & $5.99 \pm 4.06^{* * *}$ & & $5.72 \pm 4.04 * * *$ & \\
\hline Day care in the first 12 months & 16 & 12.8 & 13 & 7.1 & 9 & 7 \\
\hline Atopic dermatitis & 32 & 25.1 & 42 & 22.5 & 28 & 21.7 \\
\hline Parental atopy & 56 & 46.3 & 79 & 43.4 & 54 & 42.9 \\
\hline Smoke & 75 & 60 & 101 & 54.9 & 66 & 51.6 \\
\hline
\end{tabular}

Group A: wheezing during the first year of life; Group B: second year of life; Group C: or later; SPT: skin-prick test. **: $\mathrm{p}<0.01 ; * * *: \mathrm{p}<0.001$ (compared with group A). 


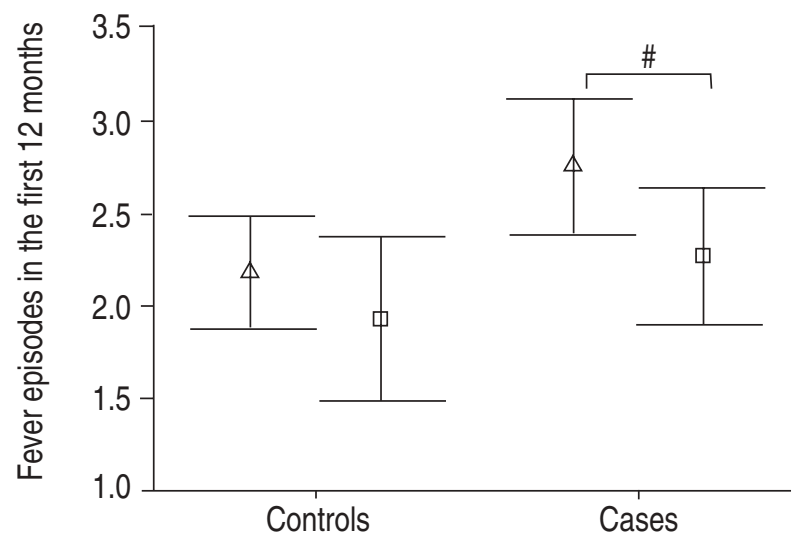

Fig. 1.-Fever episodes in the first 12 months of life by atopic sensitisation to Dermatophagoides in children with asthma (cases) and controls. Data are presented as means with $95 \%$ confidence intervals (CI). $\triangle$ : negative; $\square$ : positive. \#: odds ratio 0.84 (CI $0.74-0.95$ ), $\mathrm{p}=0.007$, for fever episode (adjusted for age, sex, daycare attendance and parental atopy).

Finally, no significant association was found between the number of fever episodes in the second year of life and atopic sensitisations both among subjects with asthma and controls.

The inverse association between atopy and fever among asthmatic children was examined after stratification of patients according to age at their first wheezing episode. Interestingly, the risk of atopy was approximately five-times lower among children with at least five fever episodes in the first year of life only in group B (OR 0.22; 95\% CI 0.07-0.65) and C (OR 0.22 ; $95 \%$ CI $0.05-0.93$ ), but not in group A (OR 0.88 ; 95\% CI 0.38-2.06) (fig. 2). An association of comparable strength was observed between fever episodes and sensitisation to Dermatophagoides: for five or more episodes OR 0.19 (95\% CI $0.05-0.67)$ in group B; for five or more episodes OR 0.22 (95\% CI 0.04-1.11) in group C.

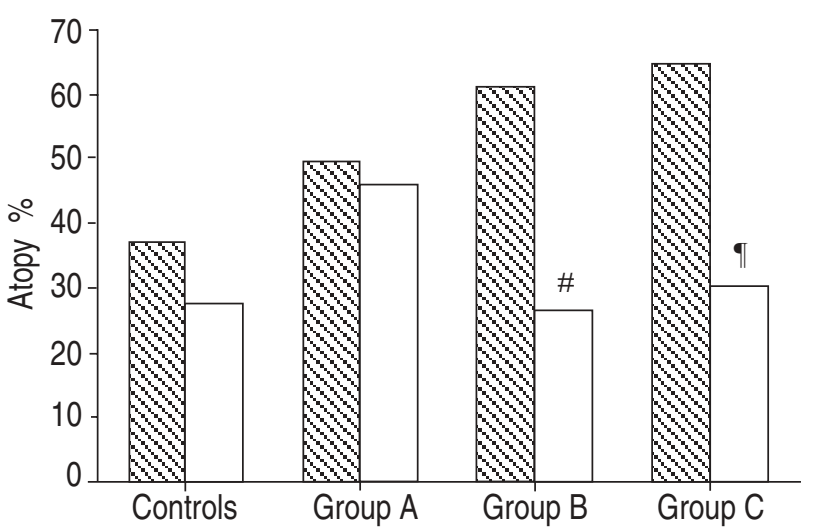

Fig. 2.-Atopic sensitisation according to number of febrile episodes in the first 12 months of life in children with asthma (wheezing during the first year of life (group $A, n=123$ ), second year of life (group B, n=174) or latter (group C, $n=119)$ ) and without asthma (controls, $\mathrm{n}=281$ ). $\mathbb{\mathbb { N }} ; \leqslant 4$ febrile episodes; $\square: \geqslant 5$ febrile episodes. ": odds ratio (OR) 0.22 (95\% confidence intervals (CI) 0.07-0.65), p<0.005; ": OR 0.22 (95\% CI 0.56-0.93), $\mathrm{p}<0.05$.

\section{Discussion}

The risk of being atopic among asthmatic children was inversely associated with the number of fever episodes in the first 12 months of life. The results suggest that children with asthma can be grouped in two categories according to their fever episodes: 1) those who start wheezing in the first year of life will develop atopic sensitisation independently from the number of fever episodes in the first year of life; 2) those who start wheezing later (after the first year of life) will have a risk of developing atopy inversely related to the number of fever episodes in the first year of life. Interestingly, within the whole asthma group only the first category is characterised by more fever episodes in the first years of life than controls. However, it is difficult to say whether having more fever episodes, probably due to infections, constitutes an independent risk factor of asthma or whether they are already the first signs of, or caused by, asthma.

Due to the cross-sectional study design, the frequency of fever episodes was retrospectively assessed by parental report, and therefore subject to a certain level of recall bias. However, it is worth noting that the mean age of the population was $6.07 \pm 2.78$ yrs lower than that of other similar studies in the literature [17]. Several confounding variables should also be accounted for in such a study. In the analysis of the data, age, sex, number of siblings, smoke, pets, daycare attendance, family history of atopic disease, breast-feeding and weaning were included, which are the variables normally considered the most important. However, other relevant factors may have an influence.

A greater number of fever episodes were found in children who started wheezing in the first year of life and the younger age of group A did not appear to be influential. In fact, parents' recall bias was excluded by a bivariate correlation procedure, which showed no association between age and number of fever episodes either in the whole population (Pearson: $r^{2}=0.00$, $\mathrm{p}=0.6$ ), or in group $\mathrm{A}$ (Pearson: $\mathrm{r}^{2}=0.00, \mathrm{p}=0.3$ ). In contrast, observational bias cannot be excluded since parents of such children may confuse wheeze episodes with fever episodes. However, children in group A reported more fever episodes also in the second year of life than children in group B (who started wheezing in the second year of life). It is unlikely that only group A parents mistake fever episodes for wheeze episodes both in the first and in the second year. These observations might point to a real difference between the groups. Finally, since asthma in infancy can be triggered by both infections and atopy, there is a risk that cross-sectional surveys might show an inverse relation between fever episodes and atopic sensitisations due to heterogeneity bias [19]. Thus, the apparent protective effect of fever against atopy may simply be due to the fact that an asthma group may be made up of different wheezing phenotypes. However, the study showed an inverse relation between fever episodes in the first year of life only in children who started wheezing after the first year of life, thus, fever episodes do not seem to trigger asthma. Moreover, there is no significant relationship between fever 
episodes and atopic sensitisations in children who started wheezing in the first year of life, where a heterogeneity bias could mainly have been expected.

This study, although carried out in a clinical setting, confirms the data obtained in an epidemiological setting in Germany [17]. The study by von Mutius et al. [17] found that fever episodes in the first 12 months of life were inversely related to later atopic sensitisation in the asthma group, but not in controls. The strength of this association was also of a similar size: (OR 0.30 for at least five episodes of fever in the present study versus 0.25 in the study by vON Mutius et al. [17]). In the present study, however, the inverse association between fever episodes and atopic sensitisation was found only among asthmatic children who started wheezing after the first year of life. Moreover, the association was largely accounted by the number of fever episodes occurring in the first 12 months of life, but not by those occurring in the second year of life.

How can fever protect from atopic sensitisation? Fever is induced by both exogenous pyrogens, like lipopolysaccaride and pyrogenic cytokines, most notably interleukin (IL)-1, IL-6 and tumour necrosis factor, that induce the production of prostaglandin $\mathrm{E}_{2}$ in the preoptic area: this mediator stimulates the neural pathways that raise body temperature [20]. It is known that the elevated body temperature enhances both the inflammatory response and the immune function. Temperature elevation appears to primarily affect the phase of recognition and sensitisation or activation of mononuclear leucocytes [21]. It has also been shown that the exposure of peripheral blood mononuclear cells to fever-range hyperthermia induces cell proliferation and enhances IFN- $\gamma$ secretion $[16,22]$ although it does not influence secretion of IL-4 [23]. These findings suggest that fever may induce a T-helper (Th)-1 response and may act as a physiological adjuvant [22]. Moreover, fever is subject to endogenous inhibitory processes via a variety of specific inhibitory mechanisms, such as the IL-1 receptor antagonist (IL-1 ra) [24]. It has recently been found that IL-1 ra blocks Th2 but not Th1 clonal proliferation in vitro and that, in the guinea pig, it reduces both bronchial hyperreactivity and pulmonary eosinophilia induced by exposure to allergens [25].

Why should fever protect from the development of atopy only children who start wheezing after the first year of life? It has recently been shown that some children with recurrent wheezing in the first year of life may develop a strong, Th2 skewed immune response [23]: therefore, it could be hypothesised that fever episodes may be ineffective in protecting children with already skewed Th2 immune responses. This may also explain the ineffectiveness of febrile episodes in the second year of life in reducing atopy. Therefore, timing of fever may also be critical, and it is conceivable that it could occur early enough to contrast the development of immune events leading to atopic sensitisation.

It has recently been debated whether infections that may protect from atopy are mainly airborne [26] or orofaecal and foodborne [27]. It would seem unlikely that respiratory infections, potentially harmful to the host, should be necessary for the postnatal maturation of a balanced immune system. On the contrary, fever serves to benefit the host and improve survival [24]. It is a phylogenetically ancient host response found in fish and conserved, with all metabolic costs, in the higher mammals including man. No systematic data were obtained on the aetiology of fever episodes in the patients, but it can be expected that most of them were due to respiratory infections. Thus, it is tempting to speculate that, at least in subjects genetically predisposed to asthma, fever induced by respiratory infections, may reduce the risk of later atopic sensitisation. Any protective effect of respiratory infections would not exclude a similar role for fever induced by gastrointestinal infections.

Finally, it has been suggested previously that both a decreased use of aspirin [28] or an increased use of paracetamol [29] may contribute to the increase of atopic diseases. In contrast, the anthroposophic lifestyle, also characterised by a reduced use of antipyretic drugs, seems to protect from atopy [30]. A role of antipyretic drugs cannot be excluded in the associations observed in this study population.

In conclusion, this study suggests that fever episodes early in life may affect the natural history of infants predisposed to asthma and highlights the need to monitor fever episodes of any kind and the use of antipyretic drugs in longitudinal studies aimed at investigating the hygiene hypothesis prospectively.

\footnotetext{
Acknowledgements. The authors would like to thank P.M. Matricardi for helpful suggestions.
}

\section{References}

1. Strachan DP. Family size, infection and atopy: the first decade of the "hygiene hypothesis". Thorax 2000; 55: s2-s10.

2. Strachan DP. Hay fever, hygiene, and household size BMJ 1989; 299: 1259-1260.

3. Kramer U, Heinrich J, Wjst M, Wichman HE. Age of entry to day nursery and allergy in later childhood. Lancet 1999; 353: 450-454.

4. Haby MM, Marks GB, Peat JK, Leeder SR. Daycare attendance before the age of two protects against atopy in preschool age children. Pediatr Pulmonol 2000; 30: 377-384.

5. Shaheen SO, Aaby P, Hall AJ, et al. Measles and atopy in Guinea-Bissau. Lancet 1996; 347: 1792-1796.

6. Matricardi PM, Rosmini F, Ferrigno L, et al. Cross sectional retrospective study of prevalence of atopy among Italian military students with antibodies against hepatitis A virus. BMJ 1997; 314: 999-1003.

7. Shirikawa T, Enomoto T, Shimazu S, Hopkin JM. The inverse association between tubercolin responses and atopic disorder. Science 1997; 275: 77-79.

8. Strannegard O, Strannegard IL. The cause of the increasing prevalence of allergy: is atopy a microbial deprivation disorder? Allergy 2001; 56: 91-102.

9. Illi S, von Mutius E, Lau S, et al. Early childhood infectious diseases and the development of asthma up 
to school age: a birth cohort study. BMJ 2001; 322 : 390-395.

10. Martinez FD. Role of viral infections in the inception of asthma and allergies during childhood: could they be protective? Thorax 1994; 49: 1189-1191.

11. Wold AE. The hygiene hypothesis revised: is the rising frequency of allergy due to changes in the intestinal flora? Allergy 1998; 53: 20-25.

12. Bodner C, Godden D, Seaton A. Family size, childhood infections and atopic diseases. Thorax 1998; 53: 28-32.

13. Paunio M, Heinonen OP, Virtanen M, Leinikki P, Patja A, Peltola H. Measles history and atopic diseases: a population-based cross-sectional study. JAMA 2000; 283: 343-346.

14. Sigurs N, Bjarnason R, Sigurbergsson F, Kjellman B. Respiratory syncitial virus in infancy is an important risk factor for asthma and allergy at age 7. Am J Respir Crit Care Med 2000; 161: 1501-1507.

15. Matricardi PM, Bonini S. High microbial turnover rate preventing atopy: a solution to inconsistencies impinging on the Hygiene hypothesis? Clin Exp Allergy 2000; 30: 1506-1510.

16. Downing JF, Martinez-Valdez H, Elizondo RS, Walker EB, Taylor MW. Hyperthermia in humans enhance interferon-gamma synthesis and alters the peripheral lymphocyte population. J Interferon Res 1988; 8: 143-150.

17. von Mutius E, Illi S, Hirsch T, Leupold W, Keil U, Weiland SK. Frequency of infections and risk of asthma, atopy and airway hyperresponsiveness in children. Eur Respir J 1999; 14: 4-11.

18. Hanifin J, Rajka G. Diagnostic features of atopic dermatitis. Acta Derm Venereol 1980; 92: 44 47.

19. Nystad W, Skrondal A, Nja F, Hetlevik O, Carlsen K-H, Magnus P. Recurrent respiratory tract infections during the first 3 years of life and atopy at school age. Allergy 1998; 53: 1189-1194.

20. Luheshi G, Rothwell N. Cytokins and fever. Int Arch Allergy Immunol 1996; 109: 301-307.
21. Roberts NJ Jr. Impact of temperature elevation on immunologic defenses. Rev Infect Dis 1991; 13: 462472.

22. Huang YH, Haegerstrand A, Frostegard J. Effects of in vitro hyperthermia on proliferative responses and lymphocyte activity. Clin Exp Immunol 1996; 103: 6166.

23. Renzi PM, Turgeon JP, Yang JP, et al. Cellular immunity is activated and a Th-2 response is associated with early wheezing in infants after bronchiolitis. Pediatrics 1997; 130: 584-593.

24. Luheshi G, Rothwell N. Cytokines and fever. Int Arch Allergy Immunol 1996; 109: 301-307.

25. Watson ML, Smith D, Bourne AD, Thompson RC, Westick J. Cytokines contribute to airway dysfunction in antigen-challenged guinea pigs: inhibition or airway hyperreactivity, pulmonary eosinophil accumulation, and tumor necrosis factor generation by pretreatment with an interleukin-1 receptor antagonist. Am J Respir Cell Mol Biol 1993; 8: 365-369.

26. Martinez FD. Role of viral infections in the inception of asthma and allergies during childhood: could they be protective? Thorax 1994; 49: 1189-1191.

27. Matricardi PM, Rosmini F, Riondino S, et al. Exposure to foodborne and orofecal microbes versus airborne viruses in relation to atopy and allergic asthma: epidemiological study. BMJ 2000; 320: 412417.

28. Varner AE, Busse WW, Lemanske RF Jr. Hypothesis: decreased use of pediatric aspirin has contributed to the increasing prevalence of childhood asthma. Ann Allergy Asthma Immunol 1998; 81: 347-351.

29. Newson RB, Shaheen SO, Chinn S, Burney PGJ. Paracetamol sales and atopic disease in children and adults: an ecological analysis. Eur Respir $J$ 2000; 16 : 817-823.

30. Alm JS, Swartz J, Lilja G, Scheynius A, Pershagen G. Atopy in children of families with anthroposophic lifestyle. Lancet 1999; 353: 1485-1488. 